\title{
Nonlinear Control System Design For Construction Robots: Estimation, Partial Linearization By Feedback And State-Dependent- Parameter Control
}

\author{
E. Shaban, K. Zied, C. J. Taylor and D. W. Seward *
}

\begin{abstract}
This work is concerned with the development of State Dependent Parameter, Proportional-IntegralPlus (SDP-PIP) control of a prototype robotic arm called Starlifter. Although the PIP controller can be interpreted as a logical extension of conventional PI/PID controllers, it exploits the power of state variable feedback methods, where the vagaries of manual tuning are replaced by pole assignment or Linear Quadratic (LQ) design. In the nonlinear case, the system is modelled using a quasilinear model structure in which the parameters vary as functions of the state variables, yielding SDP-PIP control systems in which the state feedback gains are themselves state dependent. The paper illustrates a novel partial linearization by feedback approach for handling the 'input' terms of the nonlinear model and evaluates the robustness of the method in comparison with conventional linear PIP designs.
\end{abstract}

Index Terms - PIP Control, Construction Robotics, SDP-PIP control, Partial Linearization by feed back

\section{INTRODUCTION}

I

$n$ recent years there has been a great interest in the use of robots in many areas of the construction industry, such as excavation and heavy tools deployment. However, the practical implementation of such robots is limited by various factors, including both economic and technological problems. One of the major obstacles is the difficulty of controlling heavy robots in unstructured environments such as construction sites.

In this regard, a persistent stumbling block for system developers is the achievement of adequately fast and smooth movement of hydraulically driven robot arms under automatic control. The control problem is generally made difficult by a range of factors that include highly varying loads, speeds and geometries, together with the issue of multiple hydraulic cylinders being driven by a single pump. Furthermore, the behaviour of hydraulically driven manipulators is dominated by the highly nonlinear, lightly damped dynamics of the actuators [1].

\footnotetext{
* Engineering Department, Lancaster University, LA1 4YR, UK

E. Shaban, Email: e.e.shaban@lancaster.ac.uk

K. Zied, Email: k.zied@lancaster.ac.uk

C. J. Taylor, Email: c.taylor@lancaster.ac.uk

D. W. Seward, Email: d.seward@lancaster.ac.uk
}

Such nonlinear systems present the designer with a difficult challenge, which researchers are addressing using approaches such as feedback linearization [2]; fuzzy moving sliding mode control [3]; and impedance control [4]. Although such methods show much promise, the results from field tests published to date have been mixed. For example, the success of a feedback linearization approach is highly dependent on the fidelity of the nonlinear model, and on whether the model is feedback linearisable. In the face of disturbances or parametric uncertainty, suitable performance and robustness is far from guaranteed.

Previous work on construction robots at Lancaster has focused on the Lancaster University Computerised Intelligent Excavator (LUCIE; [5]) and a prototype robotic arm called Starlifter [6]. However, control of such devices was initially based on the ubiquitous Proportional-Integral-Derivative (PID) type algorithm, tuned on-line and implemented in a rather ad hoc manner. As a result, the nonlinear joint dynamics would sometimes yield an oscillatory response. In order to maintain smooth control, therefore, previous research typically utilised a relatively slow control action. By contrast, recent research employs Proportional-Integral-Plus (PIP) design to improve the joint control and so provide smoother, more accurate movement of the excavator or robot arm [7].

Here, Non-Minimal State Space (NMSS) models are formulated, so that full state variable feedback control can be implemented directly from the measured input and output signals of the controlled process, without resort to the design and implementation of a deterministic state reconstructor (observer) or a stochastic Kalman filter: see e.g. [8-9]. The PIP controller that this approach yields can be interpreted as a logical extension of conventional PI/PID controllers. However, PIP design has numerous advantages: in particular, its structure exploits the power of state variable feedback methods, where the vagaries of manual tuning are replaced by pole assignment or Linear Quadratic (LQ) design.

It is clear from these examples, that a common strategy for the control of nonlinear systems involves 
attempting to bring the original system into a quasilinear domain, before subsequently designing an appropriate linear control algorithm [10]. Indeed, for many nonlinear systems, the essential small perturbation behaviour about an operating point can be very well approximated by simple linearised Transfer Function (TF) models and, to date, this is the approach most commonly employed in NMSS/PIP control system design.

However, one novel research area currently being investigated in order to improve PIP control in such cases, is based on State Dependent Parameter (SDP) system identification: see Young [11] and the references therein. Here, the nonlinear system is modelled using a quasi-linear model structure in which the parameters vary as functions of the state variables. In other words, SDP models are descriptions of true nonlinear systems, where the parameters are functionally dependent on other variables in the system.

The linear-like, affine structure of the SDP model means that, at each sampling instant, it can be considered as a 'frozen' linear system. This formulation is then used to design a PIP control law using linear system design strategies such as pole assignment or suboptimal LQ design. This yields SDPPIP control systems in which the state feedback gains are themselves state dependent [13].

However, not all feasible SDP model structures are controllable using this basic approach. In this regard, recent research has considered a novel partial linearization by feedback method that returns the closed-loop system to a controllable state [14].

The present paper focuses on these latest developments in SDP-PIP control, with particular reference to Starlifter [6]. As the world's first tool deployment robot designed for use in construction, its features include: a $200 \mathrm{~kg}$ lift capacity; lightweight mobile construction; hydraulic power provided by umbilical hoses to the tool-head; interchangeable tools; and operation in any orientation (e.g. at the end of a hydraulic crane boom).

For such heavy dynamic systems, it can be difficult to design appropriate open-loop experiments for data collection and subsequent model estimation. For example, system constraints limit the applicability of straightforward 'step' experiments. To help identify the SDP model for a wide range of operating conditions, the present paper combines the results from several open-loop experiments, as discussed in Section 2 below. Here, an optimisation technique is utilised to find the best 'global' model parameters.

Section 3 of the paper briefly reviews linear PIP control system design. This is followed in Section 4 by discussion of the partial linearization approach to SDP-PIP control and Section 5 by its application to Starlifter. Finally, the conclusions are presented in Section 6.

Since the focus of the present paper is the new methodological developments, only one joint of the Starlifter system will be considered for the analysis that follows, i.e. 1 degree-of-freedom. However, there is no coupling between the various joints, hence the same approach could be straightforwardly utilised for the remaining joints in the future.

\section{IDENTIFICATION AND ESTIMATION}

For a given dynamic system, an appropriate model structure needs to be identified, i.e. the most appropriate values for the triad $[n, m, \delta]$ which defines the following TF model,

$$
y_{k}=\frac{b_{\delta} z^{-\delta}+\cdots+b_{m+\delta} z^{-m-\delta}}{1+a_{1} z^{-1}+\cdots+a_{n} z^{-n}} u_{k}
$$

for which $y_{k}$ and $u_{k}$ are the output (slew joint angle) and plant input (normalised applied voltage), respectively. The two main statistical measures employed to help determine these values are the coefficient of determination $R_{T}^{2}$, based on the response error, which is a simple measure of the model fit; and the more sophisticated Young Identification Criterion (YIC), which provides a combined measure of fit and parametric efficiency, with large negative values indicating a model which sufficiently explains the data without over-parameterisation. In each case, the TF model is estimated using the Simplified Refined Instrumental Variable (SRIV) algorithm [15-16].

These statistical tools and their associated estimation algorithms have been assembled as the CAPTAIN toolbox within the MATLABC software environment (www.es.lancs.ac.uk/cres/captain). The third author can be contacted for further details about this toolbox.

\section{A. LINEAR MODELS}

For the present analysis, four data files were collected each with a different input excitation format. In each case, a first order $\mathrm{TF}$ with one numerator and one sample time delay best describes the dynamic behaviour of the system, i.e.,

$$
\hat{y}_{k}=\frac{b_{1} z^{-1}}{1+a_{1} z^{-1}} u_{k}
$$

where $z^{-1}$ is the backward shift operator, i.e. $z^{-i} y(k)=y(k-i)$. Here, $\hat{y}_{k}$ represents the estimated response of the TF model. This estimated output is related to the actual response of the system as follows, 


$$
y_{k}=\hat{y}_{k}+e_{k}
$$

where $e_{k}$ is the noise that arises from stochastic effects such as measurement noise and the effect of other input variables that are not taken into consideration. In other words, these errors are caused by the fact that the model is an approximation to reality.

One well known approach for estimating the parameters $a_{1}$ and $b_{1}$ is to minimize the following cost function,

$$
J=\sum_{k=1}^{k=N} e_{k}^{2}
$$

where $k$ is the current sample index and $N$ is the total number of samples collected. In the case of several open-loop experiments, Eq.(4) may be adjusted to take the following form,

$$
J=\sum_{1}^{M} \sum_{k=1}^{k=N} e_{k}^{2}
$$

where $M$ represents the number of data files. The selection of the initial values of the TF parameters is important, since the minimisation technique may otherwise identify the local minima rather than the desired global minima.

This problem is be overcome by application of the SRIV algorithm to one of the data sets. The estimated parameters are subsequently used as initial values for the minimisation procedure. As shown in Figure (1), the analysis suggests that estimating the parameters by optimisation averages the dynamic behaviour of the system for all the data files. The TF parameters are determined as follows: $a_{1}=-1$ and $b_{1}=0.021418$ overall, with $b_{1}=0.3677,0.8816,0.8752$ and 0.4968 , for the $1^{\text {st }}, 2^{\text {nd }}, 3^{\text {rd }}$ and $4^{\text {th }}$ data files respectively. The overall coefficient of determination $R_{T}^{2}=0.789$ for the four data files altogether.

Figure (2) shows a comparison between the model fit using the fourth data file only and the optimisation technique based on all four data files. The comparison confirms that optimisation using the entire set of data, yields TF model parameters that most appropriately describe the dynamic behaviour, regardless of the input excitation used in the open-loop experiments.

\section{B NONLINEAR MODELS}

It is assumed that the SDP model takes the same basic structure as Eq.(2), but with time varying parameters. In fact, the parameters are functionally dependent on a certain state, i.e. $a_{1, k}=f\left(\chi_{k}\right) z^{-1}$ and $b_{1, k}=f\left(\chi_{k}\right)$. In this manner, the nonlinear SDP dynamic model takes the following difference equation form,

$$
\hat{y}_{k}=-a_{1, k} \hat{y}_{k-1}+b_{1, k} u_{k-1}
$$

Analysis of experimental data from Starlifter suggests that the parameter $a_{1, k}$ is always constant $\left(a_{1}=-1\right)$. This implies that the slew joint of the robot is working as an integrator. By contrast, the parameter associated with the input is a function of the lagged input variable itself, i.e. $b_{2}=f\left(u_{k-1}\right)$. In a similar manner to the linear modelling, Figure (3) suggests that optimisation best averages the nonlinear dynamic behaviour of the system, when the analysis is simultaneously based on all four data files.

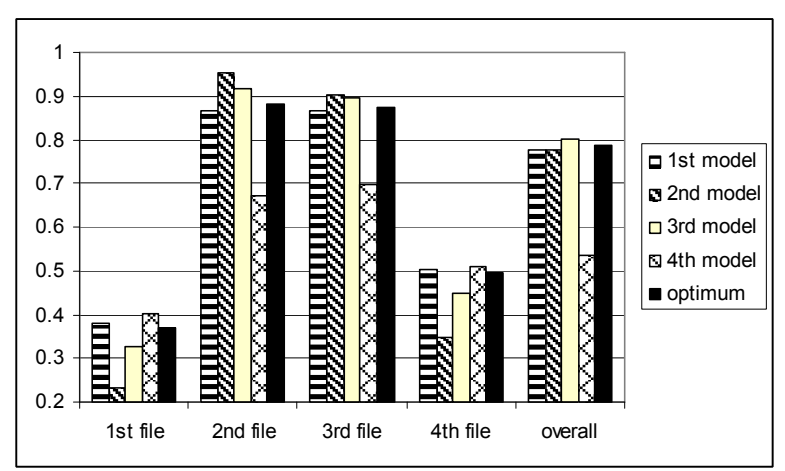

Fig. 1 Comparison of $R_{T}^{2}$ between the four TF models estimated using individual data files and using the optimization all over the data files.
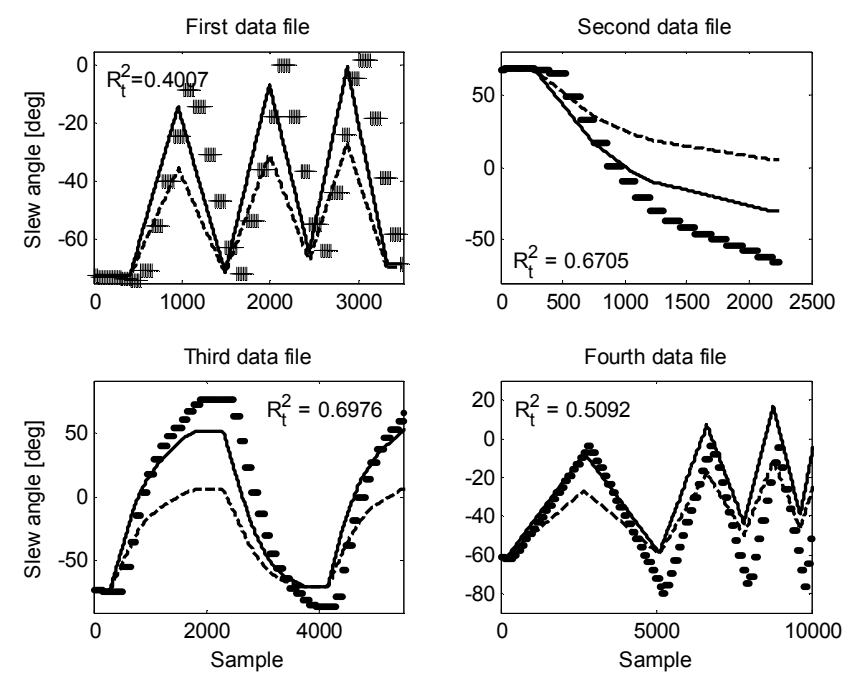

Fig. 2 Open-loop Starlifter data (dots), estimated TF model response based on estimation from the $4^{\text {th }}$ data file only (dashed) and all four data sets (solid). 


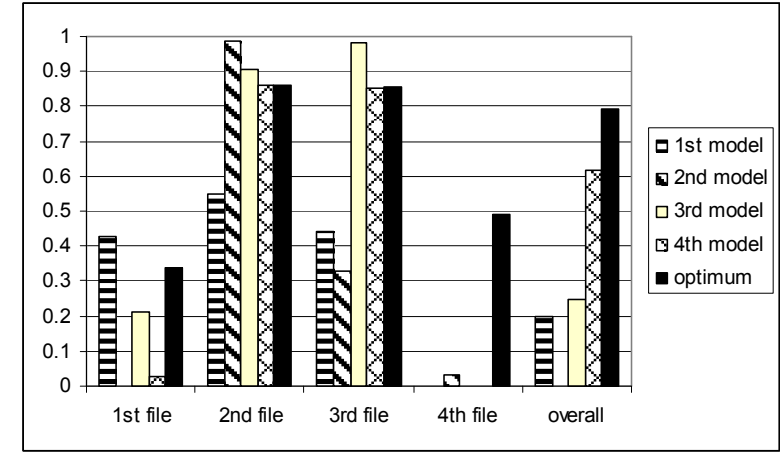

Fig. 3 Comparison of $R_{T}^{2}$ between the four SDP models estimated using individual data files and using the optimization all over the data files.

In this regard, Figure (4) compares the model fit using the fourth data file only, with that obtained using the full optimisation method. The latter approach yields an optimised $\quad b_{1}=0.0917 \times 10^{-3} u_{k-1}^{2}+0.01913$. This model yields the coefficient of determination, $R_{T}^{2}$ of $0.338,0.8603,0.8574$ and 0.4896 for the $1^{\text {st }}, 2^{\text {nd }}, 3^{\text {rd }}$ and $4^{\text {th }}$ data files respectively.
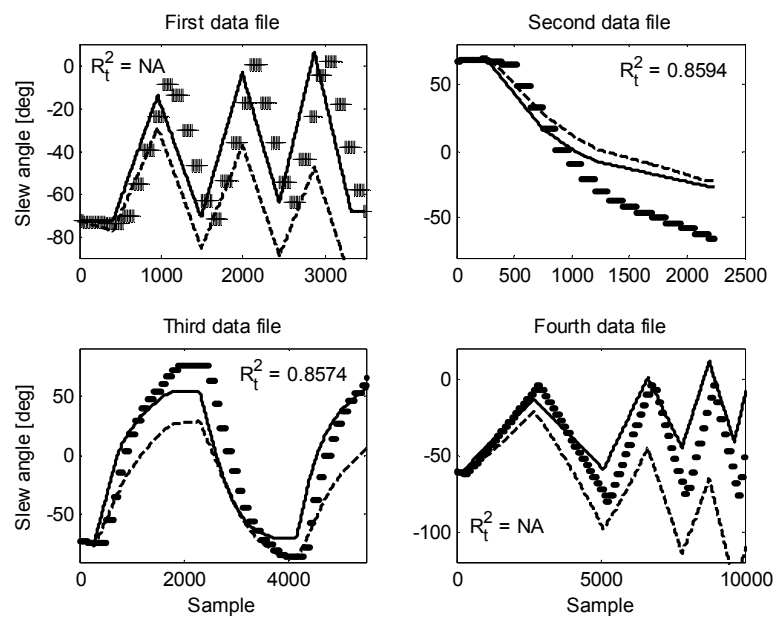

Fig. 4 Open-loop Starlifter data (dots), estimated SDP model response based on estimation from the $4^{\text {th }}$ data

file only (dashed) and all four data sets (solid).

\section{NMSS / PIP DESIGN}

The plant model considered in this work takes either the linear $\mathrm{TF}$ model form given by Eq.(2), or the nonlinear SDP form Eq.(6). Here, $a_{1}=-1$ for both cases, while $b_{1}=0.021418$ in case of the linear model and $b_{1}=0.0917 \times 10^{-3} u_{k-1}^{2}+0.01913$ for the nonlinear case.

It is easy to show that both models can be represented by the following Non-Minimal State Space (NMSS) form,

$$
\begin{aligned}
\mathbf{x}_{k+1} & =\mathbf{F} \mathbf{x}_{k}+\mathbf{g} u_{k}+\mathbf{d} r_{k} \\
y_{k} & =\mathbf{h} \mathbf{x}_{k}
\end{aligned}
$$

where $\mathbf{F}, \mathbf{g}, \mathbf{d}$ and $\mathbf{h}$ are defined by [8-9]. In the linear case, $\mathbf{F}, \mathbf{g}$ are time invariant, while in the nonlinear case, they may be time varying and detonated $\mathbf{F}_{k}, \mathbf{g}_{k}$. The $n+m$ dimensional non-minimal state vector $\mathbf{x}_{k}$ consists of the present and past sampled values of the output and the past sampled values of the input variables, i.e.,

$$
x_{k}=\left[\begin{array}{llllllll}
y_{k} & y_{k-1} & \cdots & y_{k-n+1} & u_{k-1} & \cdots & u_{k-m+1} & z_{k}
\end{array}\right]^{T}(8)
$$

Here, $z_{k}=z_{k-1}+\left(r_{k}-y_{k}\right)$ is the integral of error between the reference and the sampled output. Inherent type 1 servomechanism performance is introduced by means of this state, $z_{k}$. The control law associated with the NMSS model, Eq.(7) takes the usual State Variable Feedback (SVF) form,

$$
u_{k}=-\mathbf{v} \mathbf{x}_{k}
$$

where $\mathbf{v}=\left[\begin{array}{llllllll}f_{o} & f_{1} & \cdots & f_{n-1} & g_{1} & \cdots & g_{m-1} & -k_{I}\end{array}\right]$ is the SVF control gain vector (time invariant case).

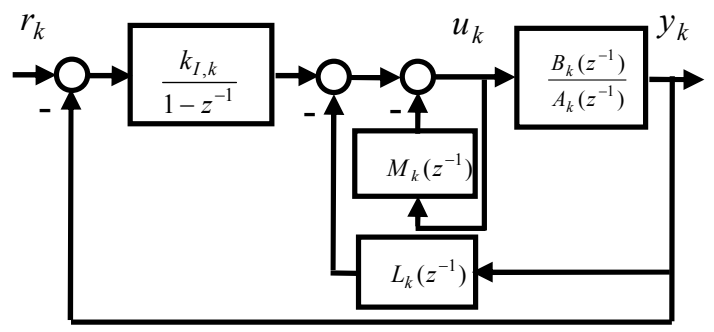

Fig. 5 PIP control block diagram.

In more conventional block diagram terms, the SVF controller, Eq.(9), can be implemented as shown in Figure (5), where it is clear that it can be considered as one particular extension of PI controller, where the PI action is, in general, enhanced by the higher order forward path and feedback compensators, $M\left(z^{-1}\right)$ and $L\left(z^{-1}\right)$, where,

$$
\begin{aligned}
L\left(z^{-1}\right) & =f_{o}+f_{1} z^{-1}+\cdots+f_{n-1} z^{-n+1} \\
M\left(z^{-1}\right) & =g_{1} z^{-1}+\cdots+g_{m-1} z^{-m+1}
\end{aligned}
$$

Note that, for the particular example given by Eq. (2), the forward path polynomial $M\left(z^{-1}\right)=0$. For the present research, the controller is obtained by optimisation in terms of a LQ cost function of the form,

$$
J=\frac{1}{2} \sum_{i=0}^{\infty}\left\{\mathbf{x}_{i}^{T} \mathbf{Q} \mathbf{x}_{i}+R u_{i}^{2}\right\}
$$

where $\mathbf{Q}$ is a diagonal state weighting matrix and $R$ is an additional scalar weight on the input. The resulting 
SVF gains are obtained recursively from the well known Algebraic Riccati Equation (ARE).

\section{PARTIAL LINEARIZATION}

Consider the system described by Eq.(6) as follows,

$$
y_{k}=y_{k-1}+\left(0.0917 \times 10^{-3} u_{k-1}^{2}+0.01913\right) u_{k-1}
$$

for which the time delay $\delta=1$. For a given nonlinear system, it is possible to find a linear input term with the same time delay, which could substitute for the input term of the nonlinear model. Then, given Eq.(12), it is possible to find a linear input term $B U_{k-1}$ such that

$$
B U_{k-1} \equiv\left(0.0917 \times 10^{-3} u_{k-1}^{2}+0.01913\right) u_{k-1}
$$

for which $B$ is constant. Theoretically, the value of $B$ could be chosen as any number. However, for the sake of robustness, the practical implementation suggests the use of a nominal value of the nonlinear input function, as shown below.

It is possible to select a constant $B$ such that minimizing the sum square of errors between the nonlinear and linear input functions, i.e.,

$$
\left(B U_{k-1}-\left(0.0917 \times 10^{-3} u_{k-1}^{2}+0.01913\right) u_{k-1}\right)^{2} \cong 0
$$

This selection of a constant $B$ would help to improve the robustness of the partially linearised system.

The second stage of the analysis is the construction of the transformation matrix $T$, which can be used for coordinate mapping as follows,

$$
\mathbf{X}_{k}=\mathbf{T} \mathbf{x}_{k}
$$

Finally the mapping described by Eq.(15) is combined with the SVF control law Eq.(9), to yield the following linearised control law,

$$
U_{k}=-\mathbf{V}_{k} \mathbf{X}_{k}=-\mathbf{V}_{k} \mathbf{T} \mathbf{x}_{k}
$$

Note that the feedback gain vector $\mathbf{V}_{k}$ has a linear input gain due to the partial linearization for the input term. Also Eq.(13) can be used for transferring back the partially linearised input $U_{k}$ to the actual input of the system $u_{k}$ by solving a $3^{\text {rd }}$ order equation. The special structure of this equation does not allow any human intervention to choose from the three potential solutions, since two of these will be imaginary, leaving just one real solution. Finally, the steps described in Eqs.(14, 15 and 16) yield the control system illustrated in figure (6).

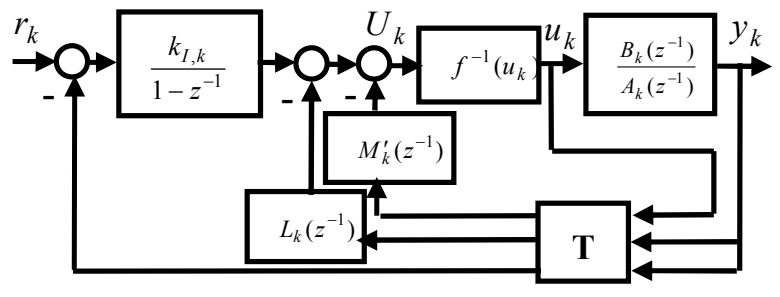

Fig.6 Linearised SDP-PIP control block diagram

\section{APPLICATION TO STARLIFTER}

Unfortunately the Starlifter robot was unavailable for the required closed-loop tests during the preparation of this paper. Therefore, to illustrate the new methodological approach, the results below are based on a simulation study.

The NMSS form for both the linear and nonlinear systems take the same structure, because of the partial linearization technique. The SDP linearised system is based on $B=0.02516386$. In this case, the NMSS form is constructed as follows,

$$
\begin{aligned}
& {\left[\begin{array}{l}
y_{k+1} \\
z_{k+1}
\end{array}\right]=\left[\begin{array}{cc}
-a_{1} & 0 \\
a_{1} & 1
\end{array}\right]\left[\begin{array}{l}
y_{k} \\
z_{k}
\end{array}\right]+\left[\begin{array}{c}
B \\
-B
\end{array}\right] u_{k}+\left[\begin{array}{l}
0 \\
1
\end{array}\right] r_{k+1}} \\
& y_{k}=\left[\begin{array}{ll}
1 & 0
\end{array}\right]\left[\begin{array}{l}
y_{k} \\
z_{k}
\end{array}\right]
\end{aligned}
$$

Experimentation suggests that setting $\mathbf{Q}=\operatorname{diag}\left[\begin{array}{ll}1 & 1\end{array}\right]$ and $R=50$ yields a suitably fast and robust PIP-LQ algorithm, for which the linearised gains are,

$$
\begin{aligned}
& f_{o}=3.21828 \\
& k_{\mathrm{I}}=0.1356
\end{aligned}
$$

The tracking test for the linearised model can be shown in Figure (7). It is important to stress here that a saturation filter was added to limit the normalised input of the slew joint between \pm 10 , together with the implementation of an appropriate incremental form to avoid the problem of integral wind-up.

The robustness of the controller to model mismatch is analysed using Monte Carlo Simulation. These results suggest that partial linearization does not significantly reduce the robustness in this case. A typical test, comparing the linear and partially linearised gains is illustrated in Figures ( 8 and 9). Here, the two controllers are applied to the nonlinear model. When using the linear gains, the system can sustain good performance if the model parameters are varied in the range $65 \%$ to $2900 \%$ of their optimised values. Using the partial linearization approach, good performance is maintained for the range $70 \%$ to $2400 \%$. 
Of course, the advantage of the nonlinear approach is the applicability of the controller to a wider range of operating conditions, once it is implemented in practice.
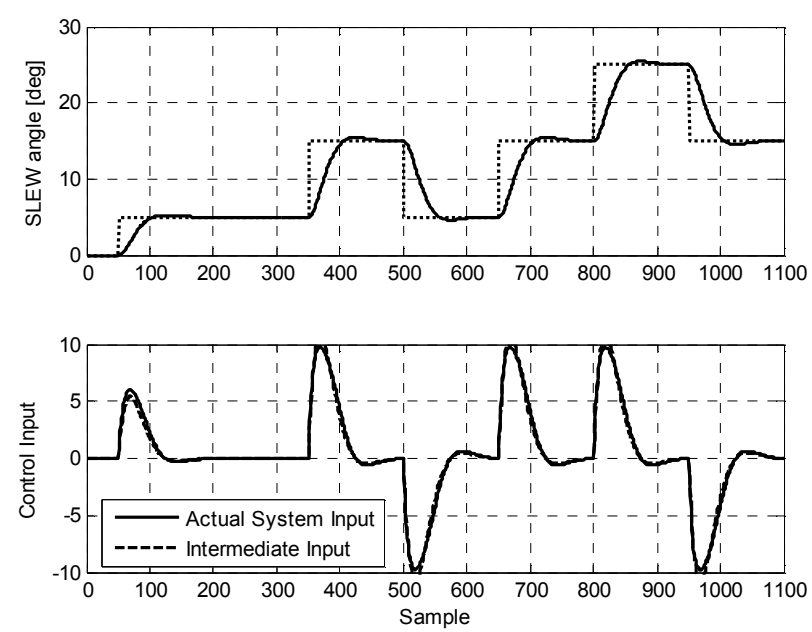

Fig. 7 Simulation response of the SLEW joint of the STARLIFTER arm.

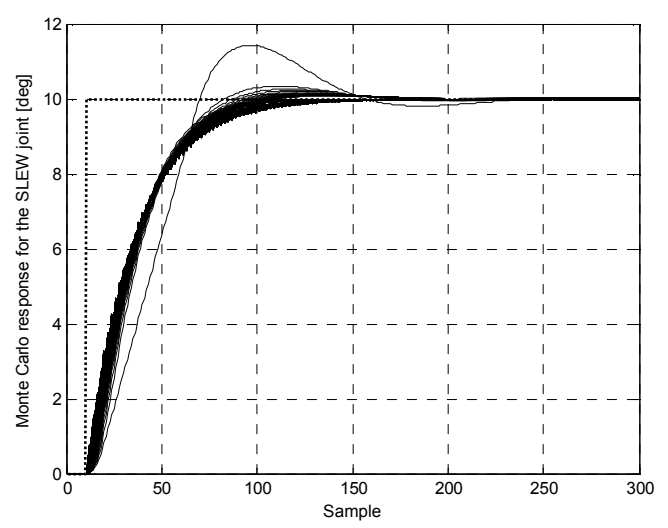

Fig. 8 Monte Carlo Simulation showing the SLEW response based on linear PIP control, using linear gains from a probability distribution in the range $65 \%$ to $2900 \%$ of their nominal values.

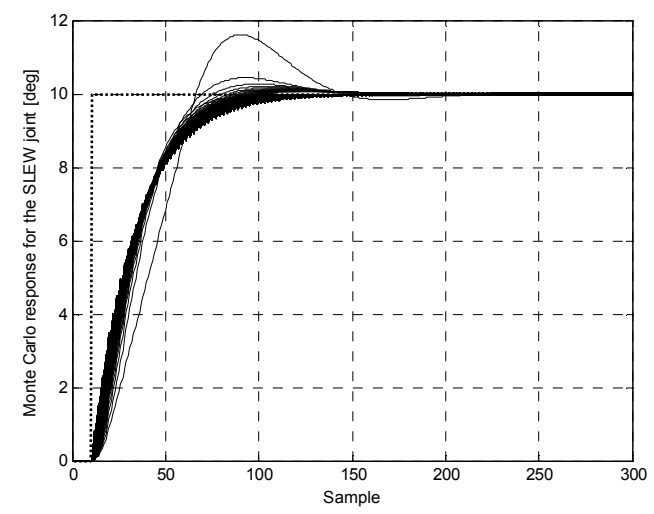

Fig. 9 Monte Carlo Simulation showing the SLEW response based on nonlinear SDP-PIP control, using linear gains from a probability distribution in the range $70 \%$ to $2400 \%$ of their nominal values.

\section{CONCLUSIONS}

This paper presents some preliminary results in the design of an automatic control system for the Starlifter robot. Although it has not been possible to implement the new controller in practice, because the robot became unavailable for use during the project, the paper has demonstrated the feasibility of the design approach using simulation.

In particular, the paper has described a novel partial linearization by feedback method for the development of State Dependent Parameter, Proportional-IntegralPlus (SDP-PIP) control systems. For the present low order example, the approach yields a fixed gain control algorithm with similar characteristics (in simulation) to a conventional linear PIP algorithm. However, in the more general case with higher order numerator terms, the final control algorithm is based on state dependent feedback gains. New examples that demonstrate the advantages of the approach in practical applications are presently being developed. These will be reported in future publications [14].

\section{ACKNOWLEDGEMENTS}

The authors are grateful for the support of the UK Engineering and Physical Sciences Research Council.

\section{REFERENCES}

[1] Merritt, E. (1976) Hydraulic Control Systems, John Wiley, New York.

[2] Nguyen, Q., Ha, Q., Rye, D. and Durrant-Whyte, H. (1999) Feedback linearization control of electro hydraulic systems of a robotic excavator, Proceedings Australian. Conference on Robotics \& Automation, Brisbane, 190-195.

[3] Ha, Q., Rye, D. and Durrant-Whyte, H. (1999) Fuzzy moving sliding mode control with application to robotic manipulators, Automatica, 35, 4, 607-616.

[4] Ha, Q., Nguyen, Q., Rye, D. and Durrant-Whyte, H. (2000) Impedance control of a hydraulic actuated robotic excavator. Journal of Automation in Construction, 9, 421-435.

[5] Bradley, D.A. and Seward, D.W. (1998) The development, control and operation of an autonomous robotic excavator, Journal of Intelligent Robotic Systems, 21, 73-97.

[6] Seward, D.W., Zied, K., Rhiehl, J. and Dolman, A. (2000) The development of a robotic system for tool deployment in hazardous environments, $17 \mathrm{th}$ Int. Symposium on Robotics in Construction, Taipei, 179-184.

[7] Gu, J., Taylor, C.J. and Seward, D. (2004) Proportional-Integral-Plus control of an intelligent excavator, Journal of Computer-Aided Civil and Infrastructure Engineering, 19, 16-27.

[8] Young, P.C., Behzadi, M.A., Wang, C.L. and Chotai, A. (1987) Direct digital and adaptive control by input-output, state variable feedback pole assignment, Int. J. of Control, 46, 1867-1881.

[9] Taylor, C.J., Chotai, A. and Young P.C. (2000) State space control system design based on nonminimal state-variable feedback : Further generalisation and unification results, Int. J. Control, 73, 1329-1345. 
[10] Isidori, A. (1995) Nonlinear control systems, 3rd Edition, Springer-Verlag, Berlin.

[11] Young, P.C. (2000) Stochastic, dynamic modelling and signal processing: time variable and state dependent parameter estimation, appears in Nonlinear and nonstationary signal processing, W.J. Fitzgerald et al. (Ed.), Cambridge University Press, 74-114.

[12] McCabe, A.P., Young P., Chotai, A. and Taylor, C.J. (2000) Proportional-Integral-Plus (PIP) control of non-linear systems, Systems Science, 26, 25-46. Publisher: OR RAN, Warszawa.

[13] Shaban, E.M., Taylor, C.J. and Chotai, A. (2004) State dependent parameter ProportionalIntegral-Plus (SDP-PIP) control of a nonlinear robot digger arm, UKACC International Conference on Control 2004, Paper-59, 6-9 September, Bath, UK.

[14] Shaban, E.M. and Taylor, C.J. (2005) State dependent parameter control with partial linearisatrion by feedback. In preparation.

[15] Young, P.C., (1984) Recursive Estimation and Time Series Analysis", Communication and Control Engineering Series, Springer-Verlag, Berlin.

[16] Young, P.C. (1991) Simplified Refined Instrumental Variable (SRIV) estimation and True Digital Control (TDC): a tutorial introduction, Proceedings of the First European Control Conference, Grenoble. 